\title{
BOUNDARY REGULARITY OF OPTIMAL TRANSPORT PATHS
}

\author{
QINGLAN XIA
}

\begin{abstract}
The optimal transport problem aims at finding an optimal way to transport a given probability measure into another. In contrast to the wellknown Monge-Kantorovich problem, the ramified optimal transportation problem aims at modeling a tree-typed branching transport network by an optimal transport path between two given probability measures. An essential feature of such a transport path is to favor group transportation in a large amount. In previous works, the author has studied the existence of optimal transport paths between probability measures as well as their interior regularity, that is away from the supports of the measures. Such an optimal transport path may be understood as a 1- dimensional rectifiable current that has boundary the difference of the 2 measures and that minimizes a suitable cost functional. In this article, we study the regularity of such an optimal transport path nearby its boundary. Motivated from observing the vein structure of a tree leaf, we show that each superlevel set of an optimal transport path is locally supported on a bi-Lipschitz graph, which is a finite union of bi-Lipschitz curves.
\end{abstract}

\section{INTRODUCTION}

This article is a continuation of previous works of the author in [15][16] about ramified optimal transportation problems. Monge-Kantorovich types of mass transport problems have been studied by many authors (see for instance, [10], [11], [7], [9], [1], [5], [14]), where the transportation between two Radon measures of equal total mass is carried out by a transport map (or a transport plan). In [15], the author provides a different model for studying a phenomenon in mass transport problems where overlapping transport is often more economical. Nearby items tend to concentrate and be carried together to their common destination far away. Such a phenomenon is very common in railway networks and in nature such as trees, circulatory systems, or lightning. Another example found in marketing also indicates this phenomenon. Goods may not be directly sold from a producer to consumers, otherwise it requires too many sales contacts. Usually there are some intermediaries (like agents, wholesales, retailers) between the producer and consumers. In our model build in [15], the transportation between two Radon measures of equal total mass is provided by a transport path rather than by a transport map. A transport path between two atomic measures is simply a weighted directed graph, while in general a transport path between two Radon measures is some vector measure whose divergence is the difference of the target measure and the initial measure in the sense of distribution. Under a suitable cost functional $\mathbf{M}_{\alpha}$ (defined below in $\S 2$ ), an optimal transport path will enjoy the ramifying structure phenomenon

2000 Mathematics Subject Classification. 90B06, 49Q20.

Key words and phrases. optimal transportation, transport path, rectifiable current, boundary regularity, branching network.

This work is supported by NSF grants DMS-0306686 and DMS-0710714. 
stated above. Results such as existence, finite total cost, length space properties, computer visualization were achieved in [15]. An application of this model to optimal urban transport networks can be found in [17]. Also, using the idea here, the author was able to provide a model for the formation of tree leaf in [18]. A big issue left in [15] is the regularity of optimal transport paths. In [16], the author studied the interior regularity of an optimal transport path. The first result was that each optimal transport path of finite $\mathbf{M}_{\alpha}$ cost is a real coefficient rectifiable 1-current. At any interior points (i.e. points away from the support of the boundary of the two given measures), the optimal transport path locally is nothing but finite union of weighted segments sharing a common endpoint and satisfying a balance equation there. In this article, we study the regularity of an optimal transport path everywhere including those boundary points.

In general, the support of an optimal transport path may not necessarily be 1 dimensional nearby its boundary, for the boundary itself may even be dense in the space, as demonstrated by letting the initial Radon measure to be the Lebesgue measure on a domain while the targeting measure be a Dirac mass. To study the boundary regularity of an optimal transport path, the main idea of our approach is to study the superlevel sets of its density function instead. Here a superlevel set of an optimal transport path means the set where the multiplicity on the transport path (viewed as a 1-dimensional rectifiable current) is no less than a given positive number. In $\S 4$ we show that each superlevel set of an optimal transport path is locally concentrated on the support of an 1-dimensional bi-Lipschitz chain, whose edges satisfy many nice properties similar to those satisfied by line segments nearby an interior point.

The main idea of the proof is based on a decomposition technique. We sketch our approach as follows. We first show in $\S 3$ that an arbitrary infinite atomic measure with a bounded $\mathbf{I}_{\alpha}$ mass (given in (3.2)) can be decomposed into two parts. The "dominant part" will contain most of the mass of the measure but its total number of elements is uniformly bounded by a number depends only on $\alpha$ and the upper bound on the $\mathbf{I}_{\alpha}$ mass of the measure. Using this decomposition, we may decompose every optimal transport path between atomic measures into the sum of two parts. The dominant part is a bi-Lipschitz chain containing those points of high multiplicity and the total number of its ending points is uniformly bounded by a number depends only on the $\mathbf{I}_{\alpha}$ mass of the boundary. This result implies that there are only finitely many possibility of the topology of the dominant part. Also, each (topological) edge of the dominant part is a bi-Lipschitz curve. In the end, we aim at decomposing an optimal transport path into two parts. Since every optimal transport path is the limit of a sequence of optimal transport path between atomic measures, we decompose locally each approximating paths into two parts as above. Both parts turn out to be weakly convergent as currents. Thus, we may decompose the optimal transport path into two parts. The dominant part contains all high density points of the path, and having the same topology as the dominant parts of approximating graphs. The desired properties of an optimal transport path then derives from the nice properties of its approximating graphs.

The idea here follows from the author's observation of the vein structure of a tree leaf. Conversely, we can use this idea to model the formation of a tree leaf, which is demonstrated in [18]. 
Since [15], [16] and the first version of this paper, there have been other interesting works on related problems such as [3], [4], [12], [19], [20] and the book [2].

In this paper, we will use the following notations:

- $X$ : a compact convex subset of a Euclidean space $\mathbb{R}^{m}$.

- $\alpha$ : a nonnegative number in $[0,1]$. Usually, $0 \leq \alpha<1$.

- $\mathcal{M}_{\Lambda}(X)$ : the space of Radon measures $\mu$ on $X$ with total mass $\mu(X)=\Lambda$.

- $\mathcal{A}_{\Lambda}(X)$ : the space of atomic measures $\mu$ on $X$ with total mass $\mu(X)=\Lambda$.

- $\mathcal{M}^{m}(X)$ : the space of Radon vector measure $\mu=\left(\mu_{1}, \mu_{2}, \cdots, \mu_{m}\right)$ on $X$.

- $\mathcal{H}^{k}$ : the $k$ dimensional Hausdorff measure.

\section{Preliminaries}

2.1. Transport paths between Radon measures. Let $X$ be a compact convex subset of a Euclidean space $\mathbb{R}^{m}$. Recall that a positive Radon measure a on $X$ is atomic if $\mathbf{a}$ is a finite sum of Dirac measures with positive multiplicities. That is

$$
\mathbf{a}=\sum_{i=1}^{k} m_{i} \delta_{x_{i}}
$$

for some integer $k \geq 1$ and some points $x_{i} \in X, m_{i}>0$ for each $i=1, \cdots, k$. The mass of the measure $\mathbf{a}$ is the number $\|\mathbf{a}\|:=\sum_{i=1}^{k} m_{i}$. For any $\Lambda>0$, let $\mathcal{A}_{\Lambda}(X)$ be the space of atomic measures on $X$ with total mass $\Lambda$.

For any $\Lambda>0$, and any two atomic measures

$$
\mathbf{a}=\sum_{i=1}^{k} m_{i} \delta_{x_{i}} \text { and } \mathbf{b}=\sum_{j=1}^{\ell} n_{j} \delta_{y_{j}}
$$

in $\mathcal{A}_{\Lambda}(X)$, a transport path from $\mathbf{a}$ to $\mathbf{b}$ is a weighted directed graph $G$ consists of a vertex set $V(G)$, a directed edge set $E(G)$ and a weight function $w: E(G) \rightarrow$ $(0,+\infty)$ such that $\left\{x_{1}, x_{2}, \ldots, x_{k}\right\} \cup\left\{y_{1}, y_{2}, \ldots, y_{\ell}\right\} \subseteq V(G)$ and for any vertex $v \in$ $V(G)$, there is a balance equation

$$
\sum_{e \in E(G), e^{-}=v} w(e)=\sum_{e \in E(G), e^{+}=v} w(e)+\left\{\begin{array}{c}
m_{i}, \text { if } v=x_{i} \text { for some } i=1, \ldots, k \\
-n_{j}, \text { if } v=y_{j} \text { for some } j=1, \ldots, \ell \\
0, \text { otherwise }
\end{array}\right.
$$

where each edge $e \in E(G)$ is a line segment from the starting endpoint $e^{-}$to the ending endpoint $e^{+}$.

Note that the balance equation (2.2) simply means the conservation of mass at each vertex. In terms of polyhedral chains, we simply have $\partial G=\mathbf{b}-\mathbf{a}$.

Let

$$
\operatorname{Path}(\mathbf{a}, \mathbf{b})
$$

be the space of all transport paths from $\mathbf{a}$ to $\mathbf{b}$. Among all paths in Path $(\mathbf{a}, \mathbf{b})$, we want to find an optimal path which allows the possibility that some parts overlap, giving higher density, in a cost efficient fashion. For this reason, we introduced the following cost functional on transport paths in [15]. 
For each transport path $G \in \operatorname{Path}(\mathbf{a}, \mathbf{b})$ as above and any $\alpha \in[0,1]$, the $\mathbf{M}_{\alpha}$ cost of $G$ is defined by

$$
\mathbf{M}_{\alpha}(G):=\sum_{e \in E(G)} w(e)^{\alpha} \text { length }(e) .
$$

Usually, we write $\mathbf{M}_{1}$ simply by $\mathbf{M}$. When $\alpha<1$, a "Y-shaped" path from two points to one point is usually more preferable than a "V-shaped" path. In general, a transport path with a ramifying structure is more efficient for $M_{\alpha}$ for $0 \leq \alpha<1$ than the one with a "linear" structure.

Another important property about transport path is given by [15, proposition 2.1]: for any transport path $G \in \operatorname{Path}(\mathbf{a}, \mathbf{b})$, there exists a transport path $\tilde{G} \in$ $\operatorname{Path}(\mathbf{a}, \mathbf{b})$ such that $\mathbf{M}_{\alpha}(\tilde{G}) \leq \mathbf{M}_{\alpha}(G)$ and $\tilde{G}$ contains no cycles. i.e. there does not exist a list of vertices $\left\{v_{1}, v_{2}, \cdots, v_{n}\right\}$ in $V(\tilde{G})$ such that $v_{1}=v_{n}$ and either $\left[v_{i}, v_{i+1}\right]$ or $\left[v_{i+1}, v_{i}\right]$ is an edge in $E(\tilde{G})$ for each $i=1,2, \cdots, n-1$. Thus, for finding an $M_{\alpha}$ minimizer in Path $(\mathbf{a}, \mathbf{b})$, we may consider only transport paths that contain no cycles.

A transport path $G \in \operatorname{Path}(\mathbf{a}, \mathbf{b})$ is called an $\alpha$-optimal transport path if it is an $M_{\alpha}$ minimizer in Path $(\mathbf{a}, \mathbf{b})$ and contains no cycles.

The following properties of an $\alpha$-optimal transport path will be used in the proof of regularity theorems.

Proposition 2.1. Suppose $G$ is an $\alpha$-optimal transport path in Path $(\mathbf{a}, \mathbf{b})$ with $0 \leq \alpha<1$, and $v$ is a vertex of $G$. Let $\left\{e_{i}\right\}_{i=1}^{d}$ be the edges in $G$ with $v$ being one of its ending points. The number $d$ is called the degree of the vertex $v$, and denoted by $\operatorname{deg}(v)$. Then

a) if $v$ is an interior vertex of $G$ (i.e. $v$ is not one of the boundary vertices $\left.\left\{x_{1}, x_{2}, \ldots, x_{k}\right\} \cup\left\{y_{1}, y_{2}, \ldots, y_{\ell}\right\}\right)$, then there exists a balance equation at $v$ :

$$
\sum_{i=1}^{d}\left[w\left(e_{i}\right)\right]^{\alpha} \vec{e}_{i}=\overrightarrow{0}
$$

where for each $i=1, \cdots, d$, the number $w\left(e_{i}\right)$ is the corresponding weight of $e_{i}$, and $\vec{e}_{i}$ is the unit directional vector of the edge $e_{i}$ from the vertex $v$ to the other endpoint of $e_{i}$.

b) The minimum angle between any two edges in $\left\{e_{i}\right\}_{i=1}^{d}$ is uniformly bounded below by

$$
\theta_{\alpha}:=\left\{\begin{array}{cc}
\text { if } 0<\alpha \leq \frac{1}{2} \\
\arccos \left(2^{\frac{\pi}{2},}, 1\right), & \text { if } \frac{1}{2}<\alpha<1 \text { or } \alpha \leq 0
\end{array} .\right.
$$

c) The degree of $v$ is bounded above by some constant $D(\alpha, m)$, depending only on $\alpha$ and the dimension $m$ of $\mathbb{R}^{m}$.

Proof. By expressing (2.3) locally in a neighborhood of the vertex $v$, the optimality of $G$ implies the equation (2.4). Then, b) follows from simple calculations using estimates achieved in [15, Example 2.1]. Then, c) follows from b).

Let $\mathcal{M}_{\Lambda}(X)$ be the space of all Radon measures on $X$ with total mass $\Lambda$. In [15], we also considered transport paths between Radon measures in $\mathcal{M}_{\Lambda}(X)$. The idea there was to approximate both Radon measures by atomic measures in $\mathcal{A}_{\Lambda}(X)$, 
and then using the transport paths of those approximating atomic measures to approximate a transport path of those Radon measures in $\mathcal{M}_{\Lambda}(X)$.

To take the limits of weighted directed graph, we view each weighted directed graph as a vector measure on $X$. Let $\mathcal{M}^{m}(X)$ be the space of Radon vector measures $\mu=\left(\mu_{1}, \mu_{2}, \cdots, \mu_{m}\right)$ on $X$. Then, each weighted directed graph $G \in$ $\operatorname{Path}(\mathbf{a}, \mathbf{b})$ determines the vector measure

$$
G=\sum_{e \in E(G)} w(e)[[e]] \in \mathcal{M}^{m}(X)
$$

on $X$, where $[[e]]$ is the vector measure $\mathcal{H}^{1}\left\llcorner e \vec{e} \in \mathcal{M}^{m}(X)\right.$ for each edge $e \in E(G)$ with unit directional vector $\vec{e}$. The balance equation (2.2) can be simplified to be a single divergence condition on $G$

$$
\operatorname{div}(G)=\mathbf{a}-\mathbf{b},
$$

in the sense of distribution. Thus,

$$
\operatorname{Path}(\mathbf{a}, \mathbf{b})=\left\{G=\sum_{e \in E(G)} w(e)[[e]] \in \mathcal{M}^{m}(X): \operatorname{div}(G)=\mathbf{a}-\mathbf{b}\right\} .
$$

Note that, when $\alpha=1$, we have

$$
\mathbf{M}(G)=\|G\|(X),
$$

where $\|G\|$ is the total variational measure of $G$.

Now, for any $\mu^{+}, \mu^{-} \in \mathcal{M}_{\Lambda}(X)$, extending definition of transport paths of atomic measures, we say a vector measure $T \in \mathcal{M}^{m}(X)$ is a transport path from $\mu^{+}$to $\mu^{-}$if there exist two sequences $\left\{\mathbf{a}_{i}\right\},\left\{\mathbf{b}_{i}\right\}$ of atomic measures in $\mathcal{A}_{\Lambda}(X)$ with a corresponding sequence of transport paths $G_{i} \in \operatorname{Path}\left(\mathbf{a}_{i}, \mathbf{b}_{i}\right)$ such that

$$
\mathbf{a}_{i} \rightarrow \mu^{+}, \mathbf{b}_{i} \rightarrow \mu^{-}, G_{i} \rightarrow T
$$

weakly as Radon measures and vector measures. The sequence of triples $\left\{\mathbf{a}_{i}, \mathbf{b}_{i}, G_{i}\right\}$ is called an approximating graph sequence for $T$. Note that for any such $T$,

$$
\operatorname{div}(T)=\mu^{+}-\mu^{-}
$$

in the sense of distributions.

Let

$$
\operatorname{Path}\left(\mu^{+}, \mu^{-}\right) \subseteq \mathcal{M}^{m}(X)
$$

be the space of all transport paths from $\mu^{+}$to $\mu^{-}$. Also, given any $\alpha \in[0,1]$, for any $T \in \operatorname{Path}\left(\mu^{+}, \mu^{-}\right)$,we define its $\mathbf{M}_{\alpha}$ cost to be

$$
\mathbf{M}_{\alpha}(T):=\inf \left(\liminf _{i \rightarrow \infty} \mathbf{M}_{\alpha}\left(G_{i}\right)\right),
$$

where the infimum is over the set of all possible approximating graph sequence $\left\{\mathbf{a}_{i}, \mathbf{b}_{i}, G_{i}\right\}$ of $T$.

The existence of $\mathbf{M}_{\alpha}$ minimizers in Path $\left(\mu^{+}, \mu^{-}\right)$with finite $\mathbf{M}_{\alpha}$ cost has been shown in [15, theroem 3.1]. In general, $\mathbf{M}_{\alpha}$ minimizers in Path $\left(\mu^{+}, \mu^{-}\right)$may not be unique. In [15, Corollary 4.2], we shown that for any approximating graph sequence $\left\{\mathbf{a}_{i}, \mathbf{b}_{i}, G_{i}\right\}$ of $T$, if each $G_{i} \in \operatorname{Path}\left(\mathbf{a}_{i}, \mathbf{b}_{i}\right)$ is an $\mathbf{M}_{\alpha}$ minimizer, then $T$ itself is also an $\mathbf{M}_{\alpha}$ minimizer in Path $\left(\mu^{+}, \mu^{-}\right)$. Such an $\mathbf{M}_{\alpha}$ minimizer is what we are interested in. 
Definition 2.2. A transport path $Y \in$ Path $\left(\mu^{+}, \mu^{-}\right)$is called an $\alpha$-optimal transport path if there exists an approximating graph sequence $\left\{\mathbf{a}_{i}, \mathbf{b}_{i}, G_{i}\right\}$ of $T$ as above such that each $G_{i} \in \operatorname{Path}\left(\mathbf{a}_{i}, \mathbf{b}_{i}\right)$ is an $\alpha$-optimal transport path.

Note that each $\alpha$-optimal transport path is automatically an $\mathbf{M}_{\alpha}$ minimizer in $\operatorname{Path}\left(\mu^{+}, \mu^{-}\right)$. We are interested in the regularity of an $\alpha$-optimal transport path

$$
T \in \operatorname{Path}\left(\mu^{+}, \mu^{-}\right)
$$

with $\alpha \in[0,1)$.

2.2. Some terminology of geometric measure theory. We first recall some terminology about rectifiable 1-currents as in [8] or [13].

Let $\Omega \subseteq \mathbb{R}^{m}$ be an open subset and $\mathcal{D}^{1}(\Omega)$ be the set of all $C^{\infty}$ differential 1-forms in $\Omega$ with compact support with the usual Fréchet topology [8]. An 1dimensional current $S$ in $\Omega$ is a continuous linear functional on $\mathcal{D}^{1}(\Omega)$. Let $\mathcal{D}_{1}(\Omega)$ denote the set of all 1-dimensional currents in $\Omega$. Motivated by Stokes' theorem, the boundary of a current $S \in \mathcal{D}_{1}(\Omega)$ is the distribution defined by

$$
\partial S(\psi):=S(d \psi)
$$

for any $C^{\infty}$ test function $\psi$ in $\Omega$ with compact support. A sequence of currents $S_{i} \in \mathcal{D}_{1}(\Omega)$ is said to be weakly convergent to another current $S \in \mathcal{D}_{1}(\Omega)$, denoted by $S_{i} \rightarrow S$, if

$$
S_{i}(\psi) \rightarrow S(\psi)
$$

for any $\psi \in \mathcal{D}^{1}(\Omega)$.

As in [13], a subset $M \subseteq \mathbb{R}^{m}$ is called (countably) 1 -rectifiable if $M=\bigcup_{i=0}^{\infty} M_{i}$, where $\mathcal{H}^{1}\left(M_{0}\right)=0$ under the 1 -dimensional Hausdorff measure $\mathcal{H}^{1}$ and each $M_{i}$, for $i=1,2, \cdots$, is a subset of an 1 -dimensional $C^{1}$ submanifold in $\mathbb{R}^{m}$. A rectifiable current $S$ is a current coming from an oriented rectifiable set with multiplicities. More precisely, $S \in \mathcal{D}_{1}(\Omega)$ is a rectifiable current if it can be expressed as

$$
S(\omega)=\int_{M}\langle\omega(x), \xi(x)\rangle \theta(x) d \mathcal{H}^{1}(x), \forall \omega \in \mathcal{D}^{1}(\Omega)
$$

where

- $M$ is a $\mathcal{H}^{1}$ measurable and 1 -rectifiable subset of $\Omega$,

- $\theta$ is a $\mathcal{H}^{1}\llcorner M$ integrable positive function, called the multiplicity function of $S$.

- $\xi: M \rightarrow \Lambda_{1}\left(\mathbb{R}^{m}\right)$ is a $\mathcal{H}^{1}$ measurable unit tangent vector field on $M$, called the orientation of $S$.

The rectifiable current $S$ described as above is often denoted by

$$
S=\underset{=}{\tau}(M, \theta, \xi) .
$$

For instance, each weighted directed graph $G \in \operatorname{Path}(\mathbf{a}, \mathbf{b})$ determines a rectifiable 1-current $G=\underset{=}{\tau}(\mathbf{G}, w, \xi)$ in $\mathbb{R}^{m}$, with $\partial G=\mathbf{b}-\mathbf{a}$ as currents. 
2.3. Transport paths viewed as one dimensional rectifiable currents. In [16], we shown that if $T$ is a transport path in Path $\left(\mu^{+}, \mu^{-}\right)$with $\mathbf{M}_{\alpha}(T)<+\infty$ for some $\alpha \in[0,1)$, then $T$ determines a rectifiable 1 -current $T=\tau(M, \theta, \xi)$ with boundary $\partial T=\mu^{+}-\mu^{-}$as currents. In this case, the $\mathbf{M}_{\alpha}$ cost of $\overline{\bar{T}}$ is simply

$$
\mathbf{M}_{\alpha}(T)=\int_{M} \theta^{\alpha}(x) d \mathcal{H}^{1}(x) .
$$

In [16], we also studied the regularity of an optimal transport path $T=\underset{=}{=}(M, \theta, \xi)$ nearby its interior points. Nevertheless, the behavior of $T$ nearby its $\overline{\bar{b}}$ oundary points (i.e. points on the support of $\partial T=\mu^{+}-\mu^{-}$) is still unclear in [16]. In this article, we will study the behavior of $T$ everywhere including at those boundary points.

The following definition is crucial for stating our boundary regularity results in section 4 .

Definition 2.3. For any $\lambda>0$, the $\lambda$-superlevel set of an $n$ dimensional rectifiable current $T=\underset{=}{\tau}(M, \theta, \xi)$ is the set

$$
T_{\lambda}:=\{p \in M: \theta(p) \geq \lambda\} .
$$

\section{Decomposition of transport paths Between atomic measures}

We first consider the decomposition of infinite atomic measures and weighted directed graphs as follows.

3.1. A decomposition of infinite atomic measures. An infinite atomic measure on $X$ is a signed measure $\mu$ on $X$ of the form

$$
\mu=\sum_{i=1}^{\infty} a_{i} \delta_{x_{i}}
$$

where $\left\{a_{i}\right\}$ is a sequence of real numbers with $\left\{\left|a_{i}\right|\right\}$ decreasing, $\sum_{i}\left|a_{i}\right|<+\infty$, and $\left\{x_{i}\right\}$ is a sequence of points in $X$. For each $\alpha \in[0,1)$, we set the $\mathbf{I}_{\alpha}$ mass of $\mu$ to be

$$
\mathbf{I}_{\alpha}(\mu):=\sum_{i=1}^{\infty}\left|a_{i}\right|^{\alpha}
$$

When $\alpha=1$, we simply write $\mathbf{I}_{1}$ by $\mathbf{I}$.

The following lemma says that a general term $\left|a_{N}\right|$ of an infinite atomic measure $\mu$ with finite $\mathbf{I}_{\alpha}$ mass can neither be too large nor be too small.

Lemma 3.1. Suppose $\mu$ is an infinite atomic measure on $X$ as in (3.1) with $\mathbf{I}_{\alpha}(\mu) \leq C<+\infty$, for some $\alpha \in[0,1)$. Then, for any $N$, we have

$$
\left|a_{N}\right|^{\alpha} \leq C / N
$$

and the remainder

$$
\sum_{i=N+1}^{\infty}\left|a_{i}\right| \leq C\left|a_{N}\right|^{1-\alpha}
$$


Proof. The inequality (3.3) follows from

$$
C \geq \sum_{i=1}^{N}\left|a_{i}\right|^{\alpha} \geq N\left|a_{N}\right|^{\alpha} .
$$

Since $\left\{\left|a_{i}\right|\right\}$ is decreasing, we have

$$
\begin{aligned}
C & \geq \sum_{i=N+1}^{\infty}\left|a_{i}\right|^{\alpha}=\left|a_{N}\right|^{\alpha} \sum_{i=N+1}^{\infty}\left(\frac{\left|a_{i}\right|}{\left|a_{N}\right|}\right)^{\alpha} \\
& \geq\left|a_{N}\right|^{\alpha} \sum_{i=N+1}^{\infty} \frac{\left|a_{i}\right|}{\left|a_{N}\right|}=\left|a_{N}\right|^{\alpha-1} \sum_{i=N+1}^{\infty}\left|a_{i}\right|,
\end{aligned}
$$

which gives the inequality (3.4).

Corollary 3.2. Suppose $\mu$ is an infinite atomic measure on $X$ in the form of (3.1) with $\mathbf{I}_{\alpha}(\mu) \leq C$ for some constant $C>0$ and $\alpha \in[0,1)$. If $\alpha>0$, then we have the estimate for the remainder

$$
\sum_{i=N+1}^{\infty}\left|a_{i}\right| \leq \frac{C^{1 / \alpha}}{N^{\frac{1-\alpha}{\alpha}}}
$$

for any $N$. If $\alpha=0$, then $a_{N}=0$ for any $N>C$.

Proof. Follows from the previous lemma.

Note that this estimate is independent of $\mu$, only depends on $C$ and $\alpha$. Using corollary 3.2 , we immediately have

Corollary 3.3. Suppose $\mu$ is an infinite atomic measure on $X$ in the form of (3.1) with $\mathbf{I}_{\alpha}(\mu) \leq C$ for some constant $C>0$ and $\alpha \in[0,1)$. For any $\epsilon>0$, one can decompose $\mu$ as the sum of

$$
\mu=\mu_{P}+\mu_{R}
$$

such that

$$
\mu_{P}=\sum_{i=1}^{N} a_{i} \delta_{x_{i}} \text { and }\left\|\mu_{R}\right\|=\sum_{i=N+1}^{\infty}\left|a_{i}\right| \leq \epsilon
$$

where $N$ is the least integer satisfying

$$
N \geq C^{\frac{1}{1-\alpha}} \epsilon^{\frac{-\alpha}{1-\alpha}} .
$$

This corollary says that an arbitrary infinite atomic measure $\mu$ with a bounded $\mathbf{I}_{\alpha}$ mass can be decomposed into two parts. The dominant part will contain most of the mass of the measure but its total number of elements is uniformly bounded by a number depends only on $\alpha$ and an upper bound $C$ for the $\mathbf{I}_{\alpha}$ mass of the measure. 


\subsection{Decomposition of transport paths. Let}

$$
\mathbf{a}=\sum_{i=1}^{k} m_{i} \delta_{x_{i}} \text { and } \mathbf{b}=\sum_{j=1}^{\ell} n_{j} \delta_{y_{j}}
$$

be any two atomic measures in $\mathcal{A}_{\Lambda}(X)$, and $G \in \operatorname{Path}(\mathbf{a}, \mathbf{b})$ be any transport path containing no cycles. Then, we may decompose $G$ as follows.

Proposition 3.4. Let $\mathbf{a}$ and $\mathbf{b}$ be any two atomic measures in $\mathcal{A}_{\Lambda}(X)$ in the form of (2.1), and $\lambda>0$. Suppose there exist natural numbers $N_{1} \leq k$ and $N_{2} \leq \ell$ such that

$$
\sum_{i=N_{1}+1}^{k} m_{i}+\sum_{j=N_{2}+1}^{\ell} n_{j}<\lambda .
$$

Then, for each transport path $G \in$ Path $(\mathbf{a}, \mathbf{b})$ containing no cycles, there exist decompositions of $\mathbf{a}, \mathbf{b}$ and $G$ (see figure 1$)$ :

$$
\mathbf{a}=\mathbf{a}_{P}+\mathbf{a}_{R}, \mathbf{b}=\mathbf{b}_{P}+\mathbf{b}_{R}, \text { and } G=P+R
$$

as atomic measures and 1-dimensional currents such that

(1) $\mathbf{a}_{P}$ is an atomic measure supported on $\left\{x_{1}, \cdots, x_{N_{1}}\right\}, \mathbf{b}_{P}$ is another atomic measure supported on $\left\{y_{1}, \cdots, y_{N_{2}}\right\}$ with the same mass as that of $\mathbf{a}_{P}$ and $P \in P$ Path $\left(\mathbf{a}_{P}, \mathbf{b}_{P}\right)$ is a transport path containing no cycles.

(2) $\mathbf{a}_{R}$ is an atomic measure supported on $\left\{x_{1}, \cdots, x_{k}\right\}, \mathbf{b}_{R}$ is an atomic measure supported on $\left\{y_{1}, \cdots, y_{l}\right\}$ and $R \in P$ Path $\left(\mathbf{a}_{R}, \mathbf{b}_{R}\right)$ is a transport path containing no cycles. Also, the mass $\left\|\mathbf{a}_{R}\right\|=\left\|\mathbf{b}_{R}\right\|<\lambda$.

(3) Moreover, the $\lambda$-superlevel set $G_{\lambda}$ of $G$ is contained in the support of $P$, where $G$ is viewed as a 1 -dimensional rectifiable current $\underset{=}{\tau}(\mathbf{G}, w, \xi)$.

Sometimes, we call $P$ in (3.5) the $\lambda$-dominant part of $G$.

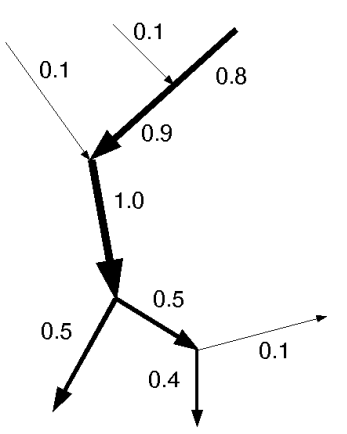

(A) $\mathrm{A}$ transport path G

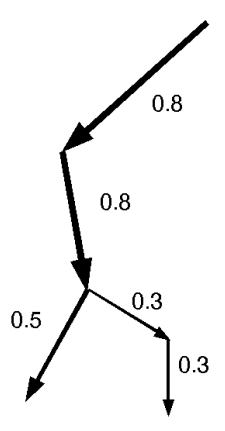

(B) The path $\mathrm{P}$

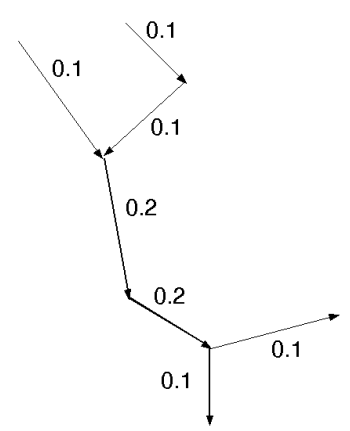

(C) The path $\mathrm{R}$

FIgURE 1. Decomposition of a transport path $G$ as sums of a dominant part $\mathrm{P}$ and a reminder part $\mathrm{R}$ with $\epsilon=0.35$. 
Proof. Suppose

$$
\mathbf{a}=\sum_{i=1}^{k} m_{i} \delta_{x_{i}} \text { and } \mathbf{b}=\sum_{j=1}^{\ell} n_{j} \delta_{y_{j}}
$$

for some $x_{i}, y_{j} \in X$ and $m_{i}, n_{j}>0$. Since $G$ is a transport path contains no cycles, for each $i=1, \cdots, k$ and $j=1, \cdots, \ell$, there exists at most one connected oriented piecewise linear curve $g_{i j}$ from $x_{i}$ to $y_{j}$, supported on $G$. The orientation of $g_{i j}$ agrees with the orientation of $G$. Each $g_{i j}$ is a rectifiable 1-current. If such curve does not exist, we set $g_{i j}$ to be 0 (as current). By [15, lemma 7.1], there exists a transport plan from $\mathbf{a}$ to $\mathbf{b}$ which is compatible with $G$. In other words, there exists a $k \times \ell$ matrix $\gamma=\left(u_{i j}\right)$ of nonnegative numbers with

$$
\sum_{j=1}^{\ell} u_{i j}=m_{i} \text { and } \sum_{i=1}^{k} u_{i j}=n_{j}
$$

such that

$$
G=\sum_{i, j} u_{i j} g_{i j}
$$

as currents. Now, we set

$$
\mathbf{a}_{P}=\sum_{i=1}^{N_{1}}\left(\sum_{j=1}^{N_{2}} u_{i j}\right) \delta_{x_{i}}, \mathbf{b}_{P}=\sum_{j=1}^{N_{2}}\left(\sum_{i=1}^{N_{1}} u_{i j}\right) \delta_{y_{j}}
$$

and

$$
P=\sum_{i=1}^{N_{1}} \sum_{j=1}^{N_{2}} u_{i j} g_{i j}
$$

Clearly, $\mathbf{a}_{P}$ and $\mathbf{b}_{P}$ have the same mass and $P \in \operatorname{Path}\left(\mathbf{a}_{P}, \mathbf{b}_{P}\right)$. Also, let

$$
\mathbf{a}_{R}=\mathbf{a}-\mathbf{a}_{P}, \mathbf{b}_{R}=\mathbf{b}-\mathbf{b}_{P} \text { and } R=G-P,
$$

then $R \in \operatorname{Path}\left(\mathbf{a}_{R}, \mathbf{b}_{R}\right)$ with

$$
\begin{aligned}
\left\|\mathbf{a}_{R}\right\| & =\left\|\mathbf{b}_{R}\right\|=\sum_{i=1}^{k} \sum_{j=1}^{\ell} u_{i j}-\sum_{i=1}^{N_{1}} \sum_{j=1}^{N_{2}} u_{i j} \\
& =\sum_{i=N_{1}+1}^{k} \sum_{j=1}^{\ell} u_{i j}+\sum_{i=1}^{N_{1}} \sum_{j=N_{2}+1}^{\ell} u_{i j} \\
& \leq \sum_{i=N_{1}+1}^{k} \sum_{j=1}^{\ell} u_{i j}+\sum_{j=N_{2}+1}^{\ell} \sum_{i=1}^{k} u_{i j} \\
& =\sum_{i=N_{1}+1}^{k} m_{i}+\sum_{j=N_{2}+1}^{\ell} n_{j}<\lambda .
\end{aligned}
$$

Let $w$ be the multiplicity function of $G$ viewed as a 1 -dimensional rectifiable current $\underset{=}{\tau}(\mathbf{G}, w, \xi)$. Then, for any $p \in \operatorname{spt}(R) \backslash \operatorname{spt}(P)$, we have

$$
w(p)=\sum_{(i, j)} u_{i j}
$$


where the summation is over all pairs $(i, j)$ such that either $i>N_{1}$ or $j>N_{2}$ and $p$ is on the support of $g_{i j}$. Thus,

$$
w(p) \leq\left\|\mathbf{a}_{R}\right\|<\lambda .
$$

This implies that the superlevel set $G_{\lambda}=\{p \in \mathbf{G}: w(p) \geq \lambda\}$ is contained in the support of $P$.

3.3. Bi-Lipschitz property of edges of the dominant part. If in addition, $G \in \operatorname{Path}(\mathbf{a}, \mathbf{b})$ is an $\alpha$-optimal transport path for some $0 \leq \alpha<1$, then we can get more information about the dominant part $P$ of $G$. From proposition 3.4, the dominant part $P$ of $G$ is still a transport path containing no cycles. A vertex $v$ of $P$ is removable if there exist only one edge (i.e. the line segment induced from $G$ ) of $P$ that flows into $v$ and only one edge of $P$ that flows out of $v$. From now on, we will only consider non-removable vertices of $P$ with the agreement that any edge of $P$ is a connected polyhedral curve between non-removable vertices of $P$, not necessarily a line segment. e.g. in figure $1 \mathrm{~b}$, the path $P$ has only three edges. Let $\left\{\Gamma_{i}\right\}_{i=1}^{K}$ be the set of all these "topological" edges of $P$ for some $K \in \mathbb{N}$. Then, as polyhedral chains, $P$ can be expressed as

$$
P=\sum_{i=1}^{K} m_{i} \Gamma_{i}
$$

for some positive numbers $m_{i}$. The following proposition says that each edge $\Gamma_{i}$ of $P$ is a bi-Lipschitz curve.

Proposition 3.5. Suppose $\mathbf{a}, \mathbf{b} \in \mathcal{A}_{\Lambda}(X)$ and $G \in$ Path $(\mathbf{a}, \mathbf{b})$ is an $\alpha$-optimal transport path with no cycles for some $0 \leq \alpha<1$. Let $P$ be the dominant part of $G$ as in (3.5). For each edge $\Gamma_{i}$ of $P$, let $\phi_{i}$ be the arc parametrization of $\Gamma_{i}$. Then $\phi_{i}$ is bi-Lipschitz with

$$
\operatorname{Lip}\left(\phi_{i}^{-1}\right) \leq \frac{\left(m_{i}\right)^{\alpha}}{\Lambda^{\alpha}-\left(\Lambda-m_{i}\right)^{\alpha}} .
$$

Proof. For any two points $\phi_{i}\left(t_{1}\right), \phi_{i}\left(t_{2}\right)$ on $\Gamma_{i}$ with $t_{1}<t_{2}$, let $L=\phi_{i}\left(\left[t_{1}, t_{2}\right]\right)$ be the part of $\Gamma_{i}$ from $\phi_{i}\left(t_{1}\right)$ to $\phi_{i}\left(t_{2}\right)$. Then $L$ is a sum of adjacent edges (or part of edges) $l_{j}$ of $G$. Let $w_{j}$ be the multiplicity of each edge $l_{j}$ in $E(G)$, then $\Lambda \geq w_{j} \geq m_{i}$, which follows from the decomposition of $G$ into $P$ and $R$ as in the proposition 3.4. Let $\left[\left[\phi_{i}\left(t_{1}\right), \phi_{i}\left(t_{2}\right)\right]\right]$ be the line segment from $\phi_{i}\left(t_{1}\right)$ to $\phi_{i}\left(t_{2}\right)$. We consider another weighted directed graph

$$
\bar{G}=G-m_{i} L+m_{i}\left[\left[\phi_{i}\left(t_{1}\right), \phi_{i}\left(t_{2}\right)\right]\right] \in \operatorname{Path}(\mathbf{a}, \mathbf{b}) .
$$

Since $G \in \operatorname{Path}(\mathbf{a}, \mathbf{b})$ is optimal, we have

$$
\mathbf{M}_{\alpha}(\bar{G}) \geq \mathbf{M}_{\alpha}(G)
$$

which yields

$$
\sum_{j}\left(w_{j}-m_{i}\right)^{\alpha} \text { length }\left(l_{j}\right)+m_{i}^{\alpha}\left|\phi_{i}\left(t_{1}\right)-\phi_{i}\left(t_{2}\right)\right| \geq \sum_{j} w_{j}^{\alpha} \operatorname{length}\left(l_{j}\right),
$$

where $|$.$| denote the Euclidean distance in \mathbb{R}^{m}$. Thus,

$$
\left|\phi_{i}\left(t_{1}\right)-\phi_{i}\left(t_{2}\right)\right| \geq \sum_{j}\left[\frac{w_{j}^{\alpha}-\left(w_{j}-m_{i}\right)^{\alpha}}{m_{i}^{\alpha}}\right] \operatorname{length}\left(l_{j}\right) \text {. }
$$


Since the function $h(x)=x^{\alpha}-(x-1)^{\alpha}$ is decreasing on $[1,+\infty)$, we have

$$
\begin{aligned}
& \left|\phi_{i}\left(t_{1}\right)-\phi_{i}\left(t_{2}\right)\right| \\
\geq & {\left[\left(\frac{\Lambda}{m_{i}}\right)^{\alpha}-\left(\frac{\Lambda}{m_{i}}-1\right)^{\alpha}\right] \sum_{j} \text { length }\left(l_{j}\right) } \\
= & {\left[\left(\frac{\Lambda}{m_{i}}\right)^{\alpha}-\left(\frac{\Lambda}{m_{i}}-1\right)^{\alpha}\right]\left|t_{1}-t_{2}\right| . }
\end{aligned}
$$

Therefore, $\phi_{i}$ is bi-Lipschitz with

$$
\operatorname{Lip}\left(\phi_{i}^{-1}\right) \leq \frac{\left(m_{i}\right)^{\alpha}}{\Lambda^{\alpha}-\left(\Lambda-m_{i}\right)^{\alpha}}
$$

Remark 3.6. From the proof of the proposition, we see that if $m_{i}+\epsilon \geq w_{j} \geq m_{i}$ for each $j$ and some $\epsilon>0$, then

$$
\operatorname{Lip}\left(\phi_{i}^{-1}\right) \leq \frac{m_{i}^{\alpha}}{\left(m_{i}+\epsilon\right)^{\alpha}-\epsilon^{\alpha}} .
$$

\section{The Boundary REgUlarity THEOREMS}

In this section, we will study the regularity of an optimal transport path nearby its boundary. More precisely, let $X$ be a convex compact subset of $\mathbb{R}^{m}$. For any Radon measures $\mu^{+}, \mu^{-} \in \mathcal{M}_{\Lambda}(X)$ of equal total mass $\Lambda>0$ and any $\alpha \in[0,1)$, let $T \in \operatorname{Path}\left(\mu^{+}, \mu^{-}\right)$be any $\alpha$-optimal transport path from $\mu^{+}$to $\mu^{-}$as in definition 2.2 with $\mathbf{M}_{\alpha}(T)<+\infty$. As stated in the $\S 2.3, T$ determines a 1 -dimensional rectifiable current $T=\underset{=}{\tau}(M, \theta, \xi)$ with boundary $\partial T=\mu^{+}-\mu^{-}$. We want to study the behavior of $T$ nearby the support of the measure $\mu^{+}-\mu^{-}$.

Here is the main difficulty of the problem. In general, the support of $T$ may not necessarily be 1 dimensional nearby its boundary, for the support of the measure $\mu^{+}-\mu^{-}$may even contain an open subset of $\mathbb{R}^{m}$. For instance, one may take $\mu^{+}$ to be some Lebesgue measure on a domain $\Omega$ and $\mu^{-}$to be some atomic measure on $\Omega$. Then, the support of $\mu^{+}-\mu^{-}$has the same dimension of $\Omega$, which is not necessarily 1 dimensional. So, the question is how to describe the behavior of $T$ when a carrying set of $T$ is possibly dense in the whole space $X$.

To study the boundary regularity of an optimal transport path, the main idea of our approach is to study its superlevel sets. This idea is motivated from observing vein structure of a tree leaf provided by the nature. In this section we show that each superlevel set of an optimal transport path is locally concentrated on the support of an 1-dimensional bi-Lipschitz chain, which is analogous to vein structures of a tree leaf.

We first clarify some terminology. For any $\lambda>0$, the $\lambda$-superlevel set of $a$ rectifiable current $T=\underset{=}{\tau}(M, \theta, \xi)$ is the set

$$
T_{\lambda}=\{x \in M: \theta(x) \geq \lambda\} .
$$

Also, a bi-Lipschitz chain $P$ is a finite sum of bi-Lipschitz curves in $X$ with real coefficient multiplicities. That is,

$$
P=\sum_{i=1}^{K} m_{i} \Gamma_{i}
$$


for some real numbers $m_{i}>0$, and some bi-Lipschitz curves $\Gamma_{i}$ with $i=1,2, \cdots, K$ in $X$. The support $\operatorname{spt}(P)$ of the bi-Lipschitz chain $P$ is the union of the image of every bi-Lipschitz curve $\Gamma_{i}$ in $X$.

Now, we state our boundary regularity theorem as follows:

Theorem 4.1. For any $\mu^{+}, \mu^{-} \in \mathcal{M}_{\Lambda}(X)$, let $T=\underset{=}{(}(M, \theta, \xi) \in \operatorname{Path}\left(\mu^{+}, \mu^{-}\right)$be any $\alpha$-optimal transport path with finite $\mathbf{M}_{\alpha}$ cost for some $0 \leq \alpha<1$. Then, for any $\epsilon>0$ and any $p \in M$, there exists an open ball neighborhood $B_{r}(p)$ about $p$ and a decomposition

$$
T\left\llcorner B_{r}(p)=P+R\right.
$$

as 1 -dimensional rectifiable currents such that

a) $P$ is a bi-Lipschitz chain in the form of (4.2).

b) $R \in$ Path $\left(\mu_{R}^{+}, \mu_{R}^{-}\right)$is an 1-dimensional rectifiable current for some Radon measures $\mu_{R}^{+}$and $\mu_{R}^{-}$with mass $\left\|\mu_{R}^{+}\right\|=\left\|\mu_{R}^{-}\right\|<\epsilon$.

c) Moreover, inside the ball $B_{r}(p)$, the $\epsilon$ - superlevel set $T_{\epsilon}$ of $T$ as defined in (4.1) is a subset of the support of the bi-Lipschitz chain P.

Proof. The proof of the theorem consists of three steps.

Step 1: uniform decomposition of approximating graphs. For each point $p \in M$, we first choose $r>0$ small enough so that the mass

$$
\|\left(\mu^{+}-\mu^{-}\right)\llcorner U \| \leq \epsilon / 4,
$$

where the open set $U=B_{r}(p) \backslash\{p\}$. By the definition 2.2 of optimal transport path, there exists a sequence of optimal transport paths $\left\{G^{(n)}\right\}$ between atomic measures such that

$$
G^{(n)} \rightarrow T, \partial G^{(n)} \rightarrow \partial T \text { and } \lim _{n \rightarrow \infty} \mathbf{M}_{\alpha}\left(G^{(n)}\right)=\mathbf{M}_{\alpha}(T)<+\infty .
$$

Thus, the sequence $\left\{\partial G^{(n)}\llcorner U\}\right.$ of signed atomic measures converges to the signed measure $\left(\mu^{+}-\mu^{-}\right)\llcorner U$. By (4.3), without losing generality, we may also require that for all $n$,

$$
\| \partial G^{(n)}\llcorner U \| \leq \epsilon / 2 .
$$

Let $S_{t}(p)$ be the sphere of center $p$ with radius $t$ in $\mathbb{R}^{m}$. For each $n$, the slicing of $G^{(n)}$ with $S_{t}(p)$ gives an atomic measure $\mu^{(n)}(t):=G^{(n)} \cap S_{t}(p)$. Then, one may check that

$$
\int_{0}^{\infty} \mathbf{I}_{\alpha}\left(\mu^{(n)}(t)\right) d t \leq \mathbf{M}_{\alpha}\left(G^{(n)}\right) \rightarrow \mathbf{M}_{\alpha}(T)<+\infty,
$$

where $\mathbf{I}_{\alpha}$ is defined in (3.2). Thus, by choosing a suitable $r$ and extracting a subsequence of $\left\{G^{(n)}\right\}$ if necessary, we can also require that

$$
\left\{\mathbf{I}_{\alpha}\left(\mu^{(n)}(r)\right)\right\}
$$

is convergent and hence uniformly bounded by some constant $C$.

Now, let $N=N(\alpha, \epsilon, C)$ be an integer such that

$$
\frac{C^{1 / \alpha}}{(N-1)^{\frac{1-\alpha}{\alpha}}} \leq \epsilon / 2 \text { if } 0<\alpha<1
$$


and $N>C+1$ if $\alpha=0$. Then, by corollary 3.2, there exists a decomposition of the atomic measure

$$
\mu^{(n)}(r)=\mu_{P}^{(n)}+\mu_{R}^{(n)}
$$

such that $\mu_{P}^{(n)}$ is an atomic measure supported on at most $N-1$ points and the total mass of $\mu_{R}^{(n)}$ is less than $\epsilon / 2$.

Let

$$
G_{n}=G^{(n)}\left\llcorner B_{r}(p)\right.
$$

be the restriction of $G_{n}$ on the ball $B_{r}(p)$. Using (4.4) and (4.6), we have

$$
\partial G_{n}=\left[\mu_{P}^{(n)}+\left(\partial G_{n}\right)(\{p\}) \delta_{p}\right]+\left[\mu_{R}^{(n)}+\partial G^{(n)}\llcorner U]\right.
$$

with

$$
\| \mu_{R}^{(n)}+\partial G^{(n)}\llcorner U \| \leq \epsilon
$$

Therefore, by the proposition 3.4, we can decompose $G_{n}$ as

$$
G_{n}=P_{n}+R_{n}
$$

as sums of polyhedral chains such that the superlevel set $\left(G_{n}\right)_{\epsilon}$ is contained in the support of $P_{n}$ and the endpoints of $P_{n}$ is at most $N$, which is independent of $n$.

Step 2: convergence of the dominant parts. Since $P_{n}$ is a directed graph with at most $N$ endpoints and contains no cycles. By a classical result in graph theory, $P_{n}$ has at most $2 N-2$ vertices and $2 N-3$ edges $\left\{\Gamma_{i}^{(n)}\right\}_{i=1}^{2 N-3}$. This fact follows from an application of the Euler formula and also a consideration of counting the total number of edges in terms of the degree of vertices. By extracting a subsequence if necessary, we may assume the vertices of $\left\{P_{n}\right\}$ are convergent. Also each $P_{n}$ can be expressed as

$$
P_{n}=\sum_{i=1}^{2 N-3} m_{i}^{(n)} \Gamma_{i}^{(n)}
$$

for some nonnegative numbers $\left\{m_{i}^{(n)}\right\}_{i=1}^{2 N-3}$. By extracting a subsequence if necessary, we may assume that for each $i=1, \cdots, N$,

$$
m_{i}^{(n)} \rightarrow m_{i}
$$

as $n \rightarrow \infty$. Now, by proposition 3.5, the arc parametrization of $\Gamma_{i}^{(n)}$ corresponds to a bi-Lipschitz map $\left\{\phi_{i}^{(n)}\right\}$ with

$$
\operatorname{Lip}\left(\left[\phi_{i}^{(n)}\right]^{-1}\right) \leq f\left(m_{i}^{(n)}\right) \rightarrow f\left(m_{i}\right)
$$

as $n \rightarrow \infty$, where $f(x)=\frac{x^{\alpha}}{\Lambda^{\alpha}-(\Lambda-x)^{\alpha}}$. Thus, if $m_{i}>0,\left\{\phi_{i}^{(n)}\right\}$ is subsequently convergent to a bi-Lipschitz map $\left\{\phi_{i}\right\}$ with

$$
\operatorname{Lip}\left(\left[\phi_{i}\right]^{-1}\right) \leq f\left(m_{i}\right) .
$$

We let $\Gamma_{i}$ be the image of $\phi_{i}$ if $m_{i}>0$. If $m_{i}=0$ i.e. $m_{i}^{(n)} \rightarrow 0$, then $m_{i}^{(n)} \Gamma_{i}^{n}$ converges to 0 as currents. In fact, since $\Gamma_{i}^{(n)}$ is bi-Lipschitz, the total length of $\Gamma_{i}^{(n)}$ is bounded above by $f\left(m_{i}^{(n)}\right)\left|u_{i}^{(n)}-v_{i}^{(n)}\right|$, where $u_{i}^{(n)}$ and $v_{i}^{(n)}$ are the endpoints 
of $\Gamma_{i}^{(n)}$ and $|$.$| is the standard Euclidean distance in \mathbb{R}^{m}$. Therefore, we have the following estimate for the total mass of $m_{i}^{(n)} \Gamma_{i}^{(n)}$, viewed as a current,

$$
\mathbf{M}\left(m_{i}^{(n)} \Gamma_{i}^{(n)}\right) \leq m_{i}^{(n)} \operatorname{length}\left(\Gamma_{i}^{(n)}\right) \leq m_{i}^{(n)} f\left(m_{i}^{(n)}\right)\left|u_{i}^{(n)}-v_{i}^{(n)}\right| \rightarrow 0 \text { as } n \rightarrow \infty .
$$

This implies $m_{i}^{(n)} \Gamma_{i}^{(n)}$ converges to zero as currents. So, let $\Gamma_{i}=0$ if $m_{i}=0$. Hence, $\left\{P_{n}\right\}$ converges to

$$
P=\sum_{i=1}^{2 N-3} m_{i} \Gamma_{i}
$$

as currents.

Step 3: properties of superlevel sets. As for $R_{n}$, each $R_{n}$ is a transport path between two Radon measures with total mass less than $\epsilon$. Thus, as real coefficient rectifiable currents, $\left\{R_{n}\right\}$ is subsequently convergent to a transport path $R$ between two Radon measures of total mass less than $\epsilon$. Thus, $G_{n}=P_{n}+R_{n} \rightarrow P+R$ as rectifiable currents. Therefore,

$$
T\left\llcorner B_{r}(p)=P+R\right.
$$

and the $\epsilon$ superlevel set

$$
T_{\epsilon}\left\llcorner B_{r}(p) \subseteq \text { the support of } P .\right.
$$

In the following corollary, we describe some nice properties of edges of the biLipschitz chain $P$.

Corollary 4.2. Let $T=\tau(M, \theta, \xi) \in$ Path $\left(\mu^{+}, \mu^{-}\right)$be any $\alpha$-optimal transport path with finite $\mathbf{M}_{\alpha}$ cost for some $0 \leq \alpha<1$. For any $\epsilon>0$ and any $p \in M$, there exists an open ball neighborhood $B_{r_{\epsilon}}(p)$ of $p$ and a bi-Lipschitz chain $Q=\sum_{i=1}^{K} m_{i} \Gamma_{i}$ such that

(1) inside the ball $B_{r_{\epsilon}}(p)$, the $\epsilon$-superlevel set $T_{\epsilon} \cap B_{r_{\epsilon}}(p)$ of $T$ is contained in the support of $Q$.

(2) The total number $K$ of edges of $Q$ is bounded above by the constant $D(\alpha, m)$ given in proposition 2.1(c).

(3) Each edge $\Gamma_{i}$ is a bi-Lipschitz curve from $p$ to a point on the sphere $S_{r_{\epsilon}}(p)$.

(4) These bi-Lipschitz curves $\Gamma_{i}$ are pairwise disjoint except at their common endpoint $p$.

(5) Moreover, the angle between any two of edges at $p$ is uniformly bounded below by $\theta_{\alpha}$ defined in (2.5).

Proof. We continue from the proof of the theorem 4.1. Without losing generality, we may assume that

$$
\left\{\Gamma_{1}, \Gamma_{2}, \cdots, \Gamma_{h}\right\}
$$

are all the $\Gamma_{i}$ 's passing through the point $p$ with multiplicity $m_{i}>0$, for some $h \leq 2 N-3$. Note that $\left\{\Gamma_{i} \backslash\{p\}\right\}_{i=1}^{h}$ must be pairwise disjoint, otherwise it will form a cycle. By adding a suitable multiple of such a cycle to $T$, one can easily find another transport path from $\mu^{+}$to $\mu^{-}$with strictly less $M_{\alpha}$ cost than $T$ has. This contradicts with the optimality of $T$. Thus, $\left\{\Gamma_{i} \backslash\{p\}\right\}_{i=1}^{h}$ are pairwise disjoint. Thus, in some small open ball neighborhood $B_{r_{\epsilon}}(p)$ of the point $p$ with 
$r_{\epsilon}<r / 2$, the chain $P$ is a finite sum of weighted bi-Lipschitz curves $\left\{\Gamma_{i}\right\}_{i=1}^{h}$. These curves are pairwise disjoint except at their common endpoint $p$. The minimum angle property follows from a similar property on optimal transport paths between atomic measures (see proposition 2.1(b)). In particular, this property implies that the total number of curves $\Gamma_{i}$ passing through $p$ is uniformly bounded above by $D(\alpha, m)$.

The following proposition indicates that locally a superlevel set of an optimal transport path is nearly the support of a bi-Lipschitz chain.

Proposition 4.3. Let $T=\underset{=}{\tau}(M, \theta, \xi) \in$ Path $\left(\mu^{+}, \mu^{-}\right)$be any $\alpha$-optimal transport path. Then, for any $\sigma_{1}>\sigma_{2}>0$ and any $p \in T_{\sigma_{1}}$, there exists an open ball neighborhood $B_{r}(p)$ of $p$ such that

$$
T_{\sigma_{1}} \cap B_{r}(p) \subseteq \text { the support of } Q_{p} \subseteq T_{\sigma_{2}} \cap B_{r}(p),
$$

where $Q_{p}=\sum_{i=1}^{K} m_{i} \Gamma_{i}$ is a bi-Lipschitz chain.

Proof. Let $\epsilon=\frac{\sigma_{1}-\sigma_{2}}{2}>0$. For any $p \in T_{\sigma_{1}}$, by theorem 4.1, there exists an open ball neighborhood $B_{r}(p)$ such that $T\left\llcorner B_{r}(p)=P+R\right.$ and

$$
T_{\epsilon}\left\llcorner B_{r}(p) \subseteq \text { the support of } P\right.
$$

for some bi-Lipschitz chain $P=\sum_{i=1}^{K} m_{i} \Gamma_{i}$. By means of $P$, we consider another bi-Lipschitz chain

$$
Q_{p}=\sum m_{i} \Gamma_{i}
$$

where the summation is over all $i=1,2, \cdots, K$ with $m_{i} \geq \frac{\sigma_{1}+\sigma_{2}}{2}$.

Claim: $T_{\sigma_{1}} \cap B_{r}(p) \subseteq$ the support of $Q_{p} \subseteq T_{\sigma_{2}} \cap B_{r}(p)$.

In fact, for each $i$, let

$$
\theta_{i}=\theta\left\llcorner\Gamma_{i},\right.
$$

where $\theta$ is the multiplicity function of the rectifiable current $T=\tau(M, \theta, \xi)$. Since the total mass of $R$ is less than $\epsilon$, we know that the range of $\overline{\bar{\theta}_{i}}$ is inside $\left[m_{i}-\epsilon, m_{i}+\epsilon\right]$. Thus, if $m_{i} \geq \frac{\sigma_{1}+\sigma_{2}}{2}$, then

$$
\theta_{i} \geq m_{i}-\epsilon \geq \frac{\sigma_{1}+\sigma_{2}}{2}-\frac{\sigma_{1}-\sigma_{2}}{2}=\sigma_{2} .
$$

Therefore,

$$
\text { the support of } Q_{p} \subseteq T_{\sigma_{2}} \cap B_{r}(p) .
$$

On the other hand, for any point $q \in T_{\sigma_{1}} \cap B_{r}(p)$, since $\sigma_{1}>\epsilon$, we have

$$
q \in T_{\epsilon}\left\llcorner B_{r}(p) \subseteq \text { the support of } P=\sum_{i=1}^{K} m_{i} \Gamma_{i} .\right.
$$

Thus, $q \in \Gamma_{i}$ for some $i$ with $\theta_{i}(q) \geq \sigma_{1}$. Hence, $m_{i} \geq \sigma_{1}-\epsilon=\frac{\sigma_{1}+\sigma_{2}}{2}$, which implies $q$ is in the support of $Q_{p}$ and

$$
T_{\sigma_{1}} \cap B_{r}(p) \subseteq \text { the support of } Q_{p} .
$$


In the end, we have the following theorem where the existence of a bi-Lipschitz graph is independent of the parameter $\epsilon$ of the superlevel sets.

Theorem 4.4. Suppose $T=\underset{=}{\tau}(M, \theta, \xi)$ is an optimal transport path with finite $\mathbf{M}_{\alpha}$ cost for some $\alpha \in[0,1)$. For any $p \in M$, there exists a bi-Lipschitz graph $\Sigma_{p}=\sum_{i=1}^{K} m_{i} \Gamma_{i}$ for some nonnegative integer $K \leq D(\alpha, m)$ such that for any positive number $\epsilon$, there exists an open ball neighborhood $B_{r_{\epsilon}}(p)$ of $p$ such that the $\epsilon$-superlevel set

$$
T_{\epsilon} \cap B_{r_{\epsilon}}(p)
$$

is supported on the support of $\Sigma_{p} \cap B_{r_{\epsilon}}(p)$. These bi-Lipschitz curves $\Gamma_{i}$ are passing through $p$ and are pairwise disjoint except at their common endpoint $p$.

Proof. For any given $p \in M$, and any $\epsilon: 0<\epsilon<\sigma=\sigma(p)$, by corollary 4.2, there exists an open ball neighborhood $B_{r_{\epsilon}}(p)$ such that

$$
T_{\epsilon} \cap B_{r_{\epsilon}}(p) \subseteq \operatorname{spt}\left(P_{\epsilon}\right)
$$

where $P_{\epsilon}=\sum_{i=1}^{k(\epsilon)} m_{i}^{(\epsilon)} \Gamma_{i}^{(\epsilon)}$ is a bi-Lipschitz chain. These curves $\Gamma_{i}^{(\epsilon)}$ 's are pairwise disjoint except at their common endpoint $\{p\}$. We may choose $r_{\epsilon}$ to be a nondecreasing function of $\epsilon \in(0, \sigma)$.

Let $k(\epsilon)$ be the total number of edges of $P_{\epsilon}$. Then as $\epsilon \rightarrow 0, k(\epsilon)$ is nondecreasing. On the other hand, this integer valued function $k(\epsilon)$ is uniformly bounded above by a constant $D(\alpha, m)$. Therefore, $k(\epsilon)$ achieves its maximum at some $\epsilon_{0} \in(0, \sigma)$. Thus, for any $\epsilon<\epsilon_{0}$, we have $k(\epsilon)=k\left(\epsilon_{0}\right)$.

Let $\tau=\min \left\{m_{i}^{\left(\epsilon_{0}\right)}: i=1,2 \cdots, k\left(\epsilon_{0}\right)\right\}>0$. Then for any $0<\epsilon<\min \left\{\tau, \epsilon_{0}\right\}$, we have

$$
\operatorname{spt}\left(P_{\epsilon_{0}}\right) \cap B_{r_{\epsilon}}(p) \subseteq T_{\tau} \cap B_{r_{\epsilon}}(p) \subseteq T_{\epsilon} \cap B_{r_{\epsilon}}(p) \subseteq \operatorname{spt}\left(P_{\epsilon}\right)
$$

by (4.8). Since $k(\epsilon)=k\left(\epsilon_{0}\right)$,

$$
\operatorname{spt}\left(P_{\epsilon_{0}}\right) \cap B_{r_{\epsilon}}(p)=\operatorname{spt}\left(P_{\epsilon}\right) \cap B_{r_{\epsilon}}(p) .
$$

Therefore, by setting $\Sigma_{p}$ to be $P_{\epsilon_{0}}$, we have

$$
T_{\epsilon} \cap B_{r_{\epsilon}}(p) \subseteq \operatorname{spt}\left(P_{\epsilon}\right) \cap B_{r_{\epsilon}}(p) \subseteq \operatorname{spt}\left(\Sigma_{p}\right) \cap B_{r_{\epsilon}}(p)
$$

as desired.

\section{REFERENCES}

[1] L. Ambrosio. Lecture notes on optimal transport problems. Mathematical aspects of evolving interfaces (Funchal, 2000), 1-52, Lecture Notes in Math., 1812, Springer, Berlin, 2003.

[2] M. Bernot; V. Caselles; J. Morel; Optimal Transportation Networks: Models and Theory. Series: Lecture Notes in Mathematics, Vol. 1955 , (2009).

[3] M. Bernot; V. Caselles; J. Morel, The structure of branched transportation networks. Calculus of Variations and Partial Differential Equations, 2008

[4] A. Brancolini, G. Buttazzo, F. Santambrogio, Path functions over Wasserstein spaces. J. Eur. Math. Soc. Vol. 8, No.3 (2006),415-434.

[5] L.A. Caffarelli; M. Feldman; R.J. McCann. Constructing optimal maps for Monge's transport problem as a limit of strictly convex costs. J. Amer. Math. Soc. 15 (2002), no. 1, 1-26

[6] G. Devillanova and S. Solimini. On the dimension of an irrigable measure. Rend. Semin. Mat. Univ. Padova 117 (2007), 1-49.

[7] L.C. Evans; W. Gangbo. Differential equations methods for the Monge-Kantorovich mass transfer problem. Mem. Amer. Math. Soc. 137 (1999), no. 653. 
[8] H. Federer. Geometric measure theory. Die Grundlehren der mathematischen Wissenschaften, Band 153 Springer-Verlag New York Inc.,New York 1969.

[9] W. Gangbo; R.J. McCann. The geometry of optimal transportation. Acta Math. 177 (1996), no. $2,113-161$.

[10] G. Monge. Mémoire sur la théorie des déblais et de remblais, Histoire de l'Académie Royale des Sciences de Paris, avec les Mémorires de Mathématique et de Physique pour la même année, pages 666-704 (1781).

[11] L. Kantorovich. On the translocation of masses. C.R. (Doklady) Acad. Sci. URSS (N.S.), 37:199-201, 1942

[12] E. Paolini and E. Stepanov. Optimal transportation networks as flat chains. Interfaces and Free Boundaries, 8 (2006), 393-436.

[13] L. Simon. Lectures on geometric measure theory. Proc. Centre Math. Anal. Australian National University. 3 (1983).

[14] C. Villani. Topics in mass transportation. AMS Graduate Studies in Math. 58 (2003).

[15] Q. Xia, Optimal paths related to transport problems. Communications in Contemporary Mathematics. Vol. 5, No. 2 (2003) 251-279.

[16] Q. Xia, Interior regularity of optimal transport paths. Calculus of Variation and PDE. Vol. 20, No. 3 (2004) 283-299.

[17] Q. Xia, An application of optimal transport paths to urban trasnport networks. Discrete and Continuous Dynmical Systems, Supplement Volume, 2005, pp 904-910.

[18] Q. Xia, The formation of a tree leaf. ESAIM Control Optim. Calc. Var. 13 (2007), no. 2, 359-377.

[19] Q. Xia. The geodesic problem in quasimetric spaces. Journal of Geometric Analysis: Volume 19, Issue2 (2009), 452-479.

[20] Q. Xia and A. Vershynina. On the transport dimension of measures. SIAM J. MATH. ANAL. Vol. 41, No. 6,(2010) pp. 2407-2430.

University of California at Davis, Department of Mathematics, Davis,CA,95616

E-mail address: qlxia@math.ucdavis.edu

$U R L:$ http://math.ucdavis.edu/ qlxia 